\title{
Implementación de la docencia en modalidad a distancia del Centro Universitario UAEM Ecatepec
}

\author{
Implementation of distance teaching at the UAEM Ecatepec University \\ Center
}

Implementação de ensino a distância no Centro Universitário UAEM

Ecatepec

Patricia Delgadillo Gómez

Universidad Autónoma del Estado de México, México

pdelgadillog@uaemex.mx

https://orcid.org/0000-0001-7871-4925

Adriana Mercedes Ruiz Reynoso

Universidad Autónoma del Estado de México, México

amruizr@uaemex.mx

https://orcid.org/0000-0003-4294-2912

Sara Lilia García Pérez

Universidad Autónoma del Estado de México, México

slgarciap@uaemex.mx

https://orcid.org/0000-0002-5395-9990

Leisdy del Carmen Gutiérrez Olmos

Universidad Autónoma del Estado de México, México

Idgutierrezo@uaemex.mx

https://orcid.org/0000-0001-5774-9635

Matilde Gómez Méndez

Universidad Autónoma del Estado de México, México

mgomezm@uaemex.mx

https://orcid.org/0000-0002-1861-5092 


\section{Resumen}

La presente investigación es de tipo descriptiva, ya que se buscó tener una medición precisa y específica acerca de la muestra seleccionada de alumnos que cursan la Licenciatura en Contaduría y Administración del Centro Universitario UAEM Ecatepec. Tiene un corte cuantitativo porque está caracterizado por recopilar, procesar y examinar información; se recabaron datos por medio de un instrumento contextualizado a la Institución en la cual se aplicó, posteriormente los resultados fueron organizados.

El objetivo principal fue analizar la viabilidad de la aplicación de la UA de Administración de las PyMES para su impartición de forma semipresencial, utilizando las TIC's como una herramienta para mejorar el proceso de enseñanza aprendizaje, e identificar si el alumno contaba con los recursos tecnológicos apropiados.

Palabras clave: enseñanza, estrategias, método, unidad de aprendizaje, virtual.

\section{Abstract}

The main objective is to analyze the feasibility of the application of the AU for SME Management for its delivery in a semi-face-to-face manner, using ICT as a tool to improve the learning teaching process; and to identify whether the student has the appropriate technological resources, as well as to analyze the skills that the teacher must have to teach the subject.

The main objective was to analyze the viability of the application of the UA of Administration of SMEs for its delivery in a blended way, using ICTs as a tool to improve the teaching-learning process, and identify if the student had the resources appropriate technology.

Keywords: Teaching, strategies, method, learning unit, virtual.

\section{Resumo}

Esta pesquisa é descritiva, visto que buscou-se uma mensuração precisa e específica sobre a amostra selecionada de alunos que cursam o Curso de Bacharelado em Ciências Contábeis e Administração do Centro Universitário UAEM Ecatepec. Possui corte quantitativo porque se caracteriza por coletar, processar e examinar informações; Os dados foram coletados por meio de um instrumento contextualizado à Instituição em que foi aplicado, posteriormente os resultados foram organizados. 


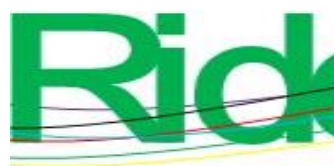

Revista Iberoamericana para la Investigación y el Desarrollo Educativo ISSN 2007 - 7467

O objetivo principal foi analisar a viabilidade da aplicação da UA de Administração de PMEs para a sua entrega de forma mista, utilizando as TIC como ferramenta para melhorar o processo de ensino-aprendizagem, e identificar se o aluno dispunha de recursos tecnológicos adequados.

Palavras-chave: ensino, estratégias, método, unidade de aprendizagem, virtual.

Fecha Recepción: Agosto 2020

Fecha Aceptación: Enero 2021

\section{Introducción}

La presente investigación es de tipo descriptiva, ya que se busca tener una medición precisa y específica acerca de la muestra seleccionada de los alumnos que cursan la licenciatura de contaduría y administración del Centro Universitario UAEM Ecatepec. Tiene un corte cuantitativo porque está caracterizado por recopilar, procesar y examinar información ya que se recabarán datos por medio de un instrumento contextualizado a la universidad en la cual se aplicó, posteriormente los resultados serán organizados.

Para este estudio se elaborará una prueba piloto, aplicada en las Licenciatura en Contaduría del Centro Universitario UAEM Ecatepec.

Teniendo en consideración que la educación es un proceso dinámico que se adapta a las necesidades de cada país y se encuentra en constante cambio, más aún en un mundo globalizado, donde la tecnología ocupa un lugar importante en el desarrollo de cada nación, los recursos, las sociedades y la interacción a través de las redes a nivel mundial. La educación es la primera en contestar al llamado tecnológico donde la formación tiene que ser permanente en un proceso de cambio e innovación en los entornos virtuales de enseñanza aprendizaje, así como el uso de las tecnologías en la Unidad de Aprendizaje (UA) de Administración de las PyMES, en la Licenciatura en Contaduría y Administración del Centro Universitario UAEM Ecatepec.

La finalidad de este estudio es buscar la factibilidad de implementar la UA Administración de las PyMES de manera semipresencial, analizando y evaluando si los alumnos cuentan con internet y computadora necesaria para su desarrollo e implementación.

Una vez que se aplicó el instrumento a los estudiantes que cursaban la asignatura como parte de esta investigación, el propósito siguiente es evaluar la viabilidad de la aplicación semipresencial, con la finalidad de hacer la petición a las autoridades pertinentes, para su aplicación en semestres posteriores. El aprendizaje colaborativo puede ser conseguido por medio del uso de estrategias colaborativas en ambientes virtuales. Se hace necesario 
contar con un soporte tecnológico para el desarrollo de estas herramientas, para que intervengan como intermediario de nuevas experiencias significativas en el ámbito educativo, produciendo el aprendizaje de conceptos, habilidades y destrezas de los alumnos. Son imprescindibles para producir aprendizajes significativos, la interacción, colaboración y diálogo afines a los que en la actualidad se demandan como: la creatividad, capacidad de análisis, reflexión, pensamiento, razonamiento, abstracción, resolución, planteamiento de alternativas, entre otras.

\section{Entornos virtuales}

El aprendizaje y la educación juegan un papel muy importante en el desarrollo social y son uno de los motores de su proceso de desarrollo. Es por ello que el proceso de enseñanza debe adaptarse siempre a las características personales que lo configuran.

Por otro lado, en los últimos años estamos viviendo una verdadera revolución tecnológica, que ha cambiado nuestros hábitos de vida y afectado nuestro entorno, en ocasiones saturado o abrumado por todas estas tecnologías. (García, 2001)

La educación a distancia también conocida en México como en línea, virtual o e-lerning, ha tenido gran impacto en la educación superior, de diferentes formas, complejidades que impactan en la pedagogía, la tecnología, la organización y la economía, con recursos de aprendizaje evolutivos, sistemas de evaluación progresivos, siendo un proceso que se encuentra en aplicación y mejora, analizando marcos normativos para que este modelo educativo tenga un impacto importante en su quehacer diario, en la investigación y vinculación con la sociedad.

Este modelo es una realidad universitaria en México, vinculando la pedagogía, la tecnología, las instituciones, docentes, tutores, especialistas y alumnos, tomándose como una modalidad compleja, con paradigmas nuevos y visiones intelectuales diferentes, potencializando las realidades o bien contraponiéndose, en ambiente académico o de trabajo, buscando como objetivo comprender nuevas realidades con diseños y desarrollos de modelos educativos evolutivos, sumándose instituciones, programas, modelos de oferta en las distintas áreas, buscando componentes de calidad y equidad.

El mundo sufre transformación progresiva, actualmente se destaca por el uso de las nuevas tecnologías de la información y comunicación, el individuo tiene que ser capaz de prosperar y renovarse en este mundo de continuos cambios, los sistemas educativos también tienen que estarse renovando.

Actualmente el sistema educativo de la UAEM brinda al individuo la oportunidad de prepararse, de adaptarse a los constantes cambios que se viven día a día, para ello la 


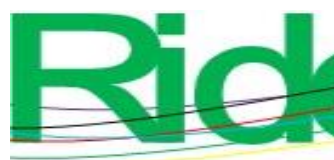

Revista Iberoamericana para la Investigación y el Desarrollo Educativo ISSN 2007 - 7467

Universidad va a la vanguardia tomando en consideración los procesos de cambio, la cultura y evolución histórica, modificando los contenidos, jerarquías, métodos, buscando que el hombre no únicamente conozca, sino que pase al saber ser.

En la siguiente tabla se muestran las características de cada una de las modalidades que ofrece la institución que son modalidad abierta, modalidad a distancia y modalidad mixta, modalidad presencial.

Tabla 1. Modalidades de enseñanza de la UAEM

\begin{tabular}{|c|c|}
\hline Modelo & Descripción \\
\hline $\begin{array}{l}\text { Estudio } \\
\text { independiente guiado } \\
\text { (modalidad abierta) }\end{array}$ & $\begin{array}{l}\text { El estudiante se encontrará solo y aislado al momento de leer } \\
\text { los materiales, aportando ideas personales, preguntas y juicios; } \\
\text { involucrándolo para lograr un interés en la materia o problema } \\
\text { en cuestión. }\end{array}$ \\
\hline $\begin{array}{l}\text { 2. Aula remota } \\
\text { (modalidad a } \\
\text { distancia) }\end{array}$ & $\begin{array}{l}\text { Se basa en el uso de las TIC para reproducir en distancia lo que } \\
\text { normalmente ocurre en un salón de clases presencial, llamado } \\
\text { por Miller "aula distribuida" (2004), generalmente se manejan } \\
\text { tecnologías que consienten la transmisión sincrónica (en } \\
\text { tiempo real, en vivo y espontáneas) de audio y/o video. Se } \\
\text { alcanzan sólo sitios establecidos por la institución y no por los } \\
\text { estudiantes. }\end{array}$ \\
\hline $\begin{array}{l}\text { 3. Modelo } \\
\text { interactivo } \\
\text { basado en las } \\
\text { TIC (modalidad a } \\
\text { distancia) }\end{array}$ & $\begin{array}{l}\text { Utiliza tecnologías de Internet para el acceso a los materiales y } \\
\text { para el contacto entre asesores académicos y estudiantes, en } \\
\text { interacción sincrónica y/o asincrónica. También conocido } \\
\text { como educación a distancia basada en redes o "en línea", la } \\
\text { interacción entre el profesor y el estudiante se incrementan, } \\
\text { mas no asegura, el conocimiento por parte de los estudiantes. }\end{array}$ \\
\hline $\begin{array}{l}\text { 4. Modelo híbrido } \\
\text { (modalidad mixta) }\end{array}$ & $\begin{array}{l}\text { Se mezclan educación presencial y a distancia de tal manera } \\
\text { que ambas experiencias de aprendizaje son imprescindibles } \\
\text { para el éxito de los objetivos de la misma. Se requiere que } \\
\text { trabajen juntas de manera lógica como las partes de una } \\
\text { máquina. El modelo basado en las TIC se utiliza para la entrega } \\
\text { de contenidos, simulaciones, el desarrollo de actividades } \\
\text { colaborativas, el proceso de retroalimentación y el de } \\
\text { interacción. El modelo presencial se utiliza para sensibilizar al } \\
\text { estudiante en los contenidos, practicar, discutir los retos de } \\
\text { estos conocimientos y habilidades en el ámbito laboral y } \\
\text { asegurar el compromiso social. }\end{array}$ \\
\hline $\begin{array}{l}\text { 5. Modelo presencial } \\
\text { apoyado con } \\
\text { tecnología } \\
\text { (modalidad } \\
\text { presencial) }\end{array}$ & $\begin{array}{l}\text { Modelos más que mixtos son presenciales apoyados con el uso } \\
\text { de las TIC. Continuo entre la educación presencial tradicional } \\
\text { y la educación a distancia. No obstante, en los extremos de este } \\
\text { espectro encontraremos educación presencial con muy poco } \\
\text { apoyo a distancia y educación a distancia con muy poca } \\
\text { presencia. }\end{array}$ \\
\hline
\end{tabular}




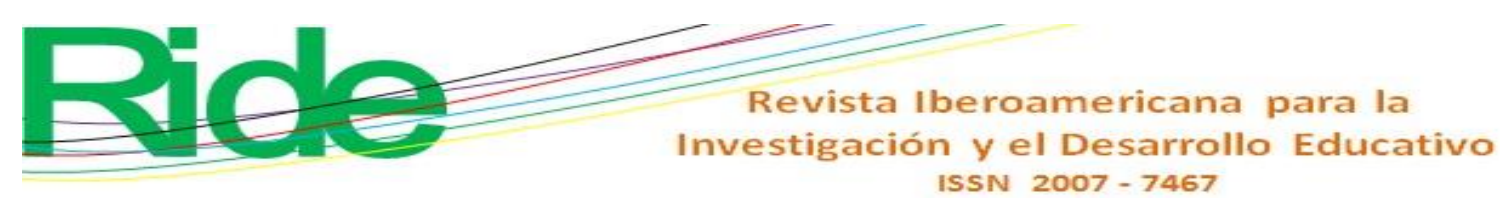

Fuente: Elaboración propia extraído de Universidad Autónoma del Estado de México.

(2009). Plan General de Desarrollo 2009-2021. Toluca, México.

http://web.uaemex.mx/abogado/doc/0057\%20LinEdCont.pdf.

La Universidad Autónoma del Estado de México ofrece diferentes opciones de enseñanza a quienes desean formarse profesionalmente en alguna de las carreras profesionales que ofrece, así como cada uno de los Centro Universitarios adscritos, el alumno puede elegir la opción más adecuada para su formación profesional con base en sus intereses, disposición, recursos económicos y tecnológicos.

Para estudiar de manera virtual (no escolarizada) los estudiantes deben ser emprendedores, creativos e innovadores, tener gusto por la tecnología y contar con los recursos tecnológicos necesarios que les permitan estudiar e incluso alternar con un trabajo, desde una perspectiva autodidacta.

\section{Metodología}

Se desarrolló una investigación cuantitativa por que se aplicó un cuestionario para recabar datos, analizarlos y comprobar la hipótesis, de tipo documental a través de la consulta de libros, revistas, memorias, registros, entre otros. Es exploratoria porque se explica el propósito de recabar información en un solo momento. Transeccional o transversal ya que los datos se recabaron en un solo momento siendo los datos de tipo descriptivo para reconocer, ubicar y definir situaciones particulares; fundamentar la hipótesis, para recoger ideas o sugerencias que permitan afinar la metodología, estrategias, etcétera, formulando con mayor exactitud el esquema de investigación definitivo.

Identificar la viabilidad de la implementación de la asignatura de Administración de las PyMES a distancia para las Licenciaturas de Contaduría y Administración, si cuentan los alumnos con la infraestructura necesaria para cursar la UA de aprendizaje mencionada.

El total de la población es de 28 alumnos de Contaduría y Administración, inscritos en el periodo primavera 2019, se aplicó el cuestionario para realizar la prueba piloto correspondiente, buscando la confiabilidad y validez de ésta, aplicándolo a la totalidad de los alumnos para obtener resultados confiables y representativos.

En el Centro Universitario UAEM Ecatepec se tiene una matrícula de estudiantes inscritos de 1440, que corresponden a las licenciaturas de Contaduría, Administración, Informática Administrativa, Ingeniería en Computación, Psicología y Derecho; para esta 
investigación se utilizará a los alumnos inscritos en cuarto semestre de la Licenciatura de Contaduría y Administración, que cursan la asignatura de Administración de las PyMES.

\section{Resultados}

Figura 1. Programa Educativo

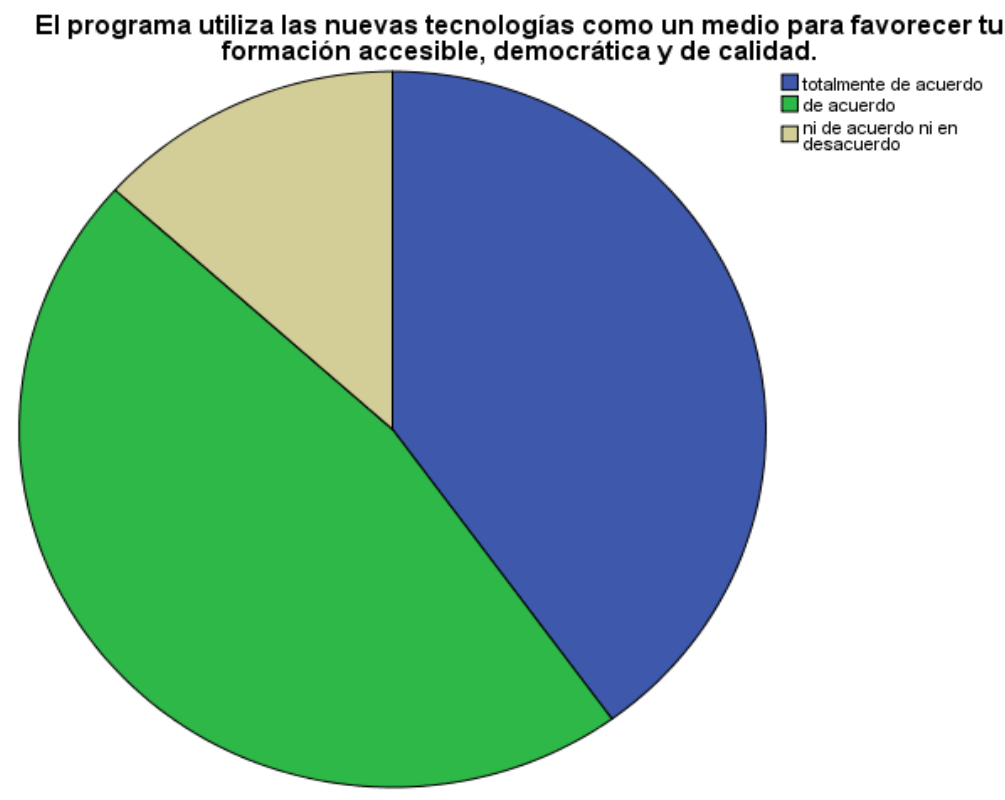

Fuente: Elaboración propia

La sumativa de los totalmente de acuerdo y los de acuerdo es de $86.7 \%$ de los alumnos que consideran que las nuevas tecnologías son un medio accesible que favorecen su formación de manera democrática y de calidad, y solo el $13.3 \%$ no está ni de acuerdo ni en desacuerdo. 


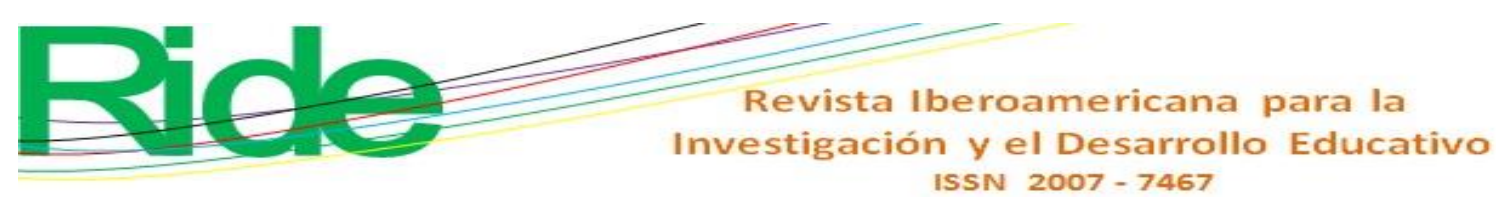

Figura 2. Funcionalidad del programa educativo

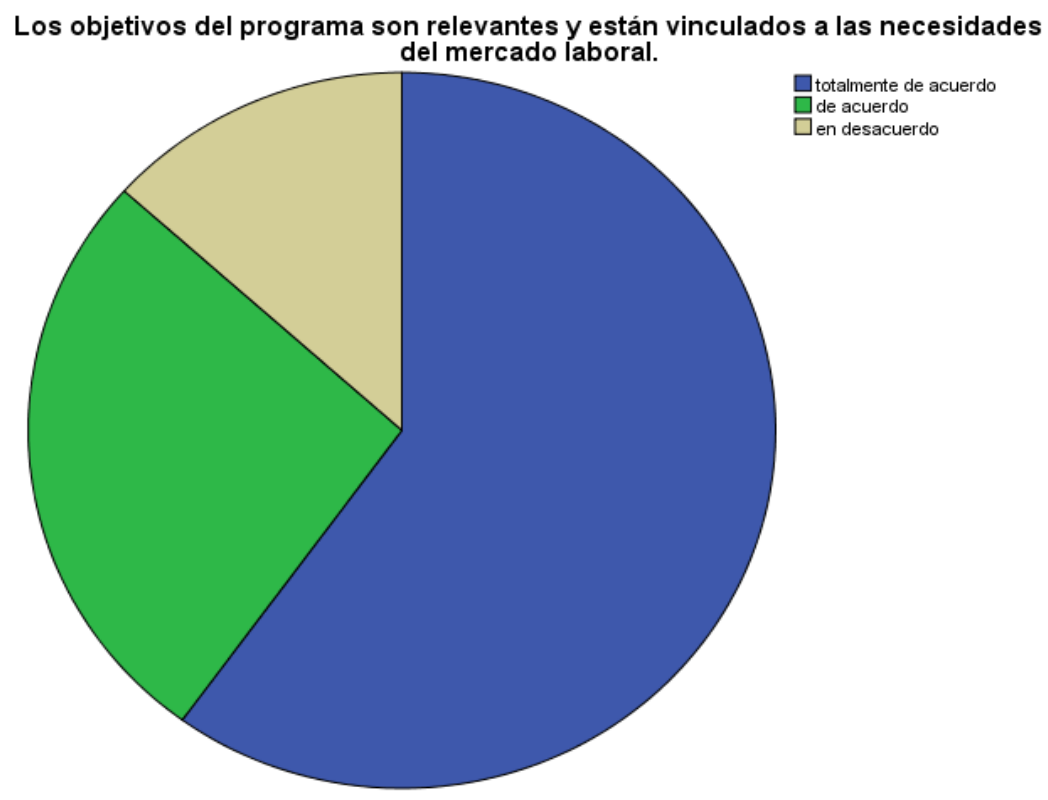

Fuente: Elaboración propia

Únicamente el $13.3 \%$ de los encuestados considera que los objetivos del programa educativo no son relevantes ni están vinculados a las necesidades del mercado laboral, contra el $86.7 \%$ que lo considera pertinente.

Figura 3. Estrategias del programa educativo

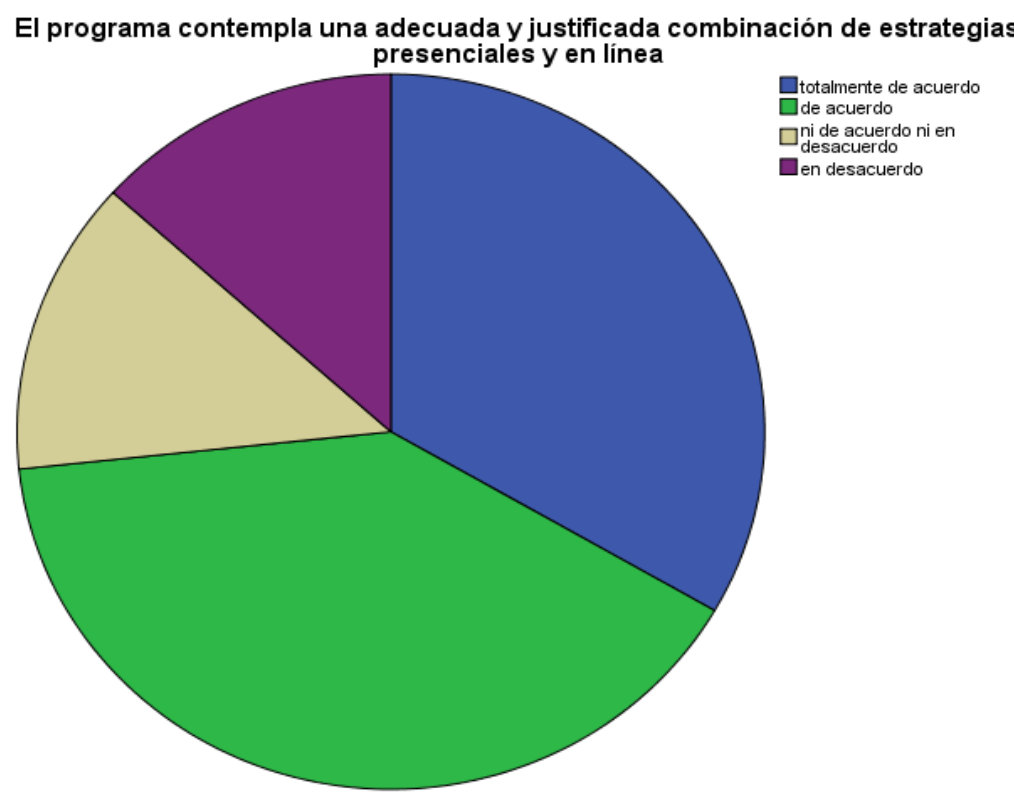

Fuente: Elaboración propia

Los estudiantes consideran que el programa contempla las estrategias de manera presencial y en línea estando totalmente de acuerdo y de acuerdo con 73.3\%, los restantes 


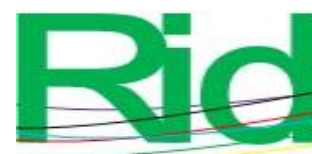

Revista Iberoamericana para la Investigación y el Desarrollo Educativo ISSN 2007 - 7467

consideran estar ni de acuerdo ni en desacuerdo y ni en desacuerdo con un $26.6 \%$, de la muestra ninguno contesto estar totalmente en desacuerdo.

Figura 4. Alcances del programa educativo

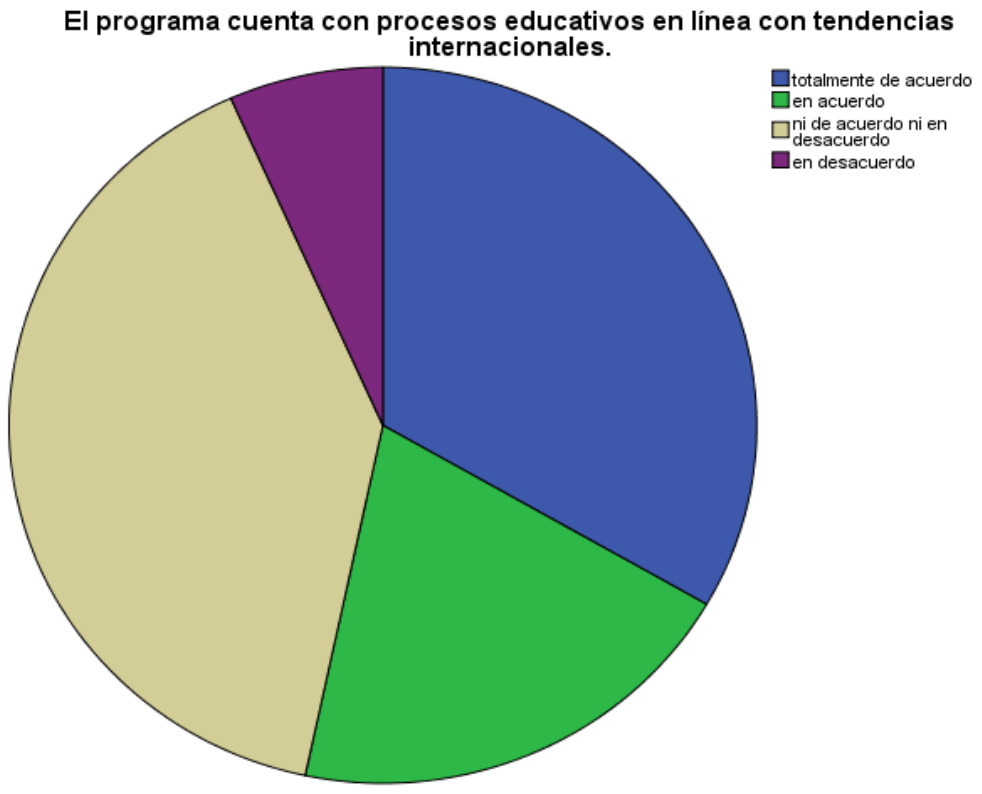

Fuente: Elaboración propia

Más de la mitad de los alumnos encuestados consideran que el programa educativo tiene una atendencia internacional con un $53.3 \%$, siendo la minoría el $6.7 \%$ que está en desacuerdo.

Figura 5. Nivel de competencia del programa

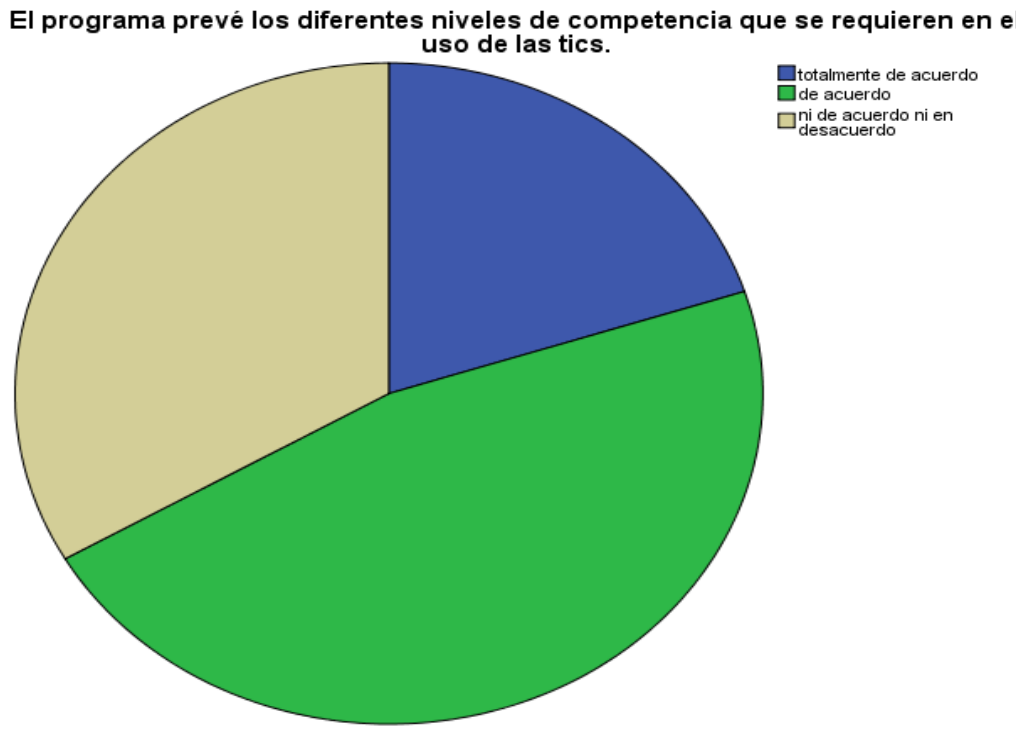

Fuente: Elaboración propia 
El $66.7 \%$ de los estudiantes considera que el programa cuenta con las competencias que se requieren para el uso de las TIC's.

Figura 6. Estrategias de aprendizaje en el programa de estudios

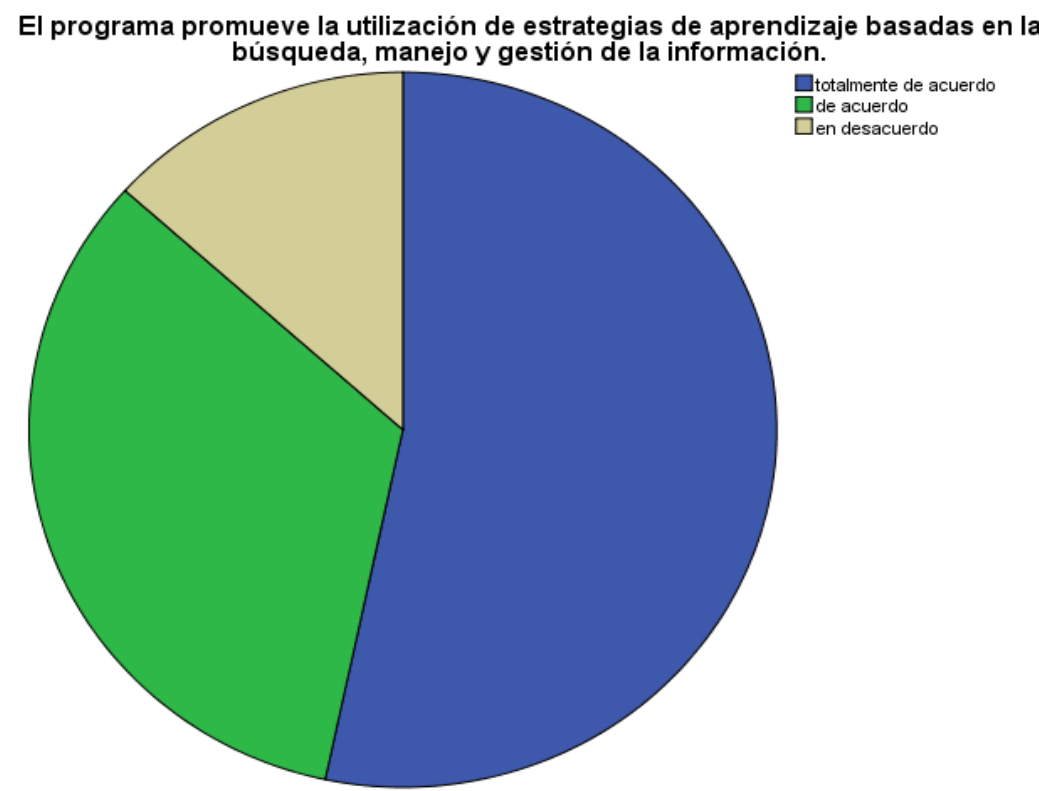

Fuente: Elaboración propia

Los encuestados consideran estar totalmente de acuerdo y de acuerdo con un $86.7 \%$ en que el programa promueve la utilización de estrategias de aprendizaje basado en la información, en su manejo, búsqueda y gestión para transformarla en conocimiento y sólo el $13.3 \%$ está en desacuerdo.

Figura 7. Estrategias innovadoras en el programa de estudios

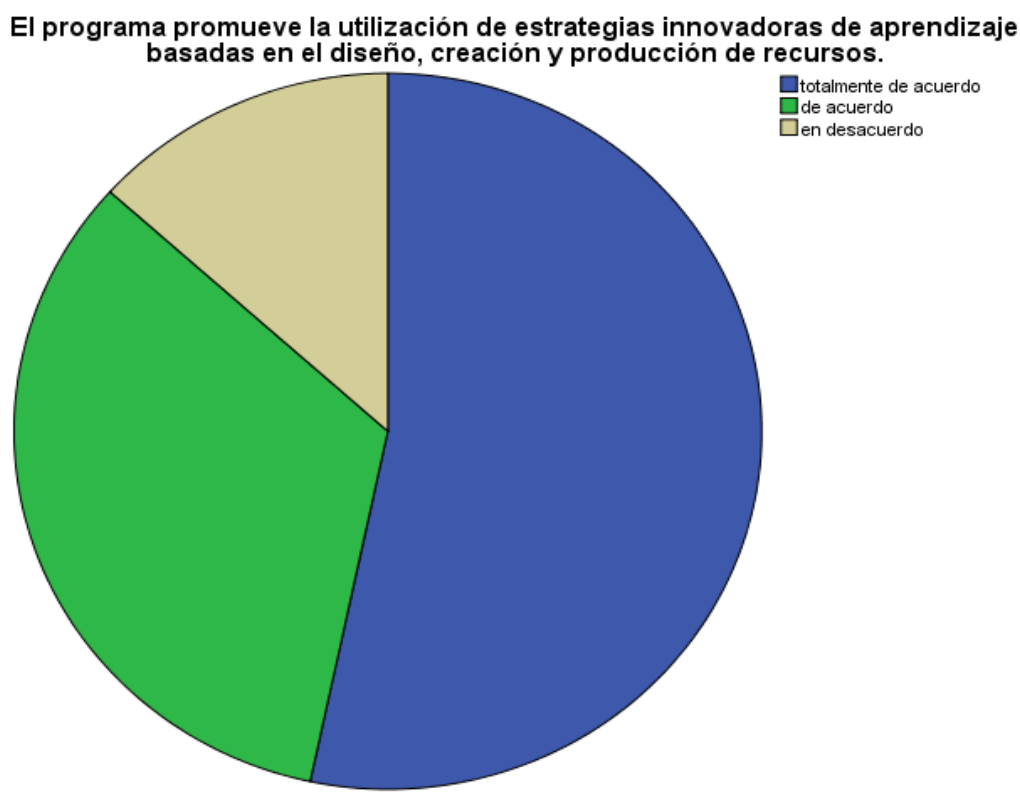

Fuente: Elaboración propia 


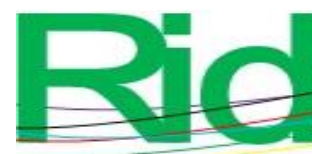

Revista Iberoamericana para la

Los encuestados consideran que el programa promueve la utilización, creación y producción de recursos innovadores de aprendizaje con un $86.7 \%$, mientras que el $13.3 \%$ está en desacuerdo.

Figura 8. Personalización del ambiente de aprendizaje

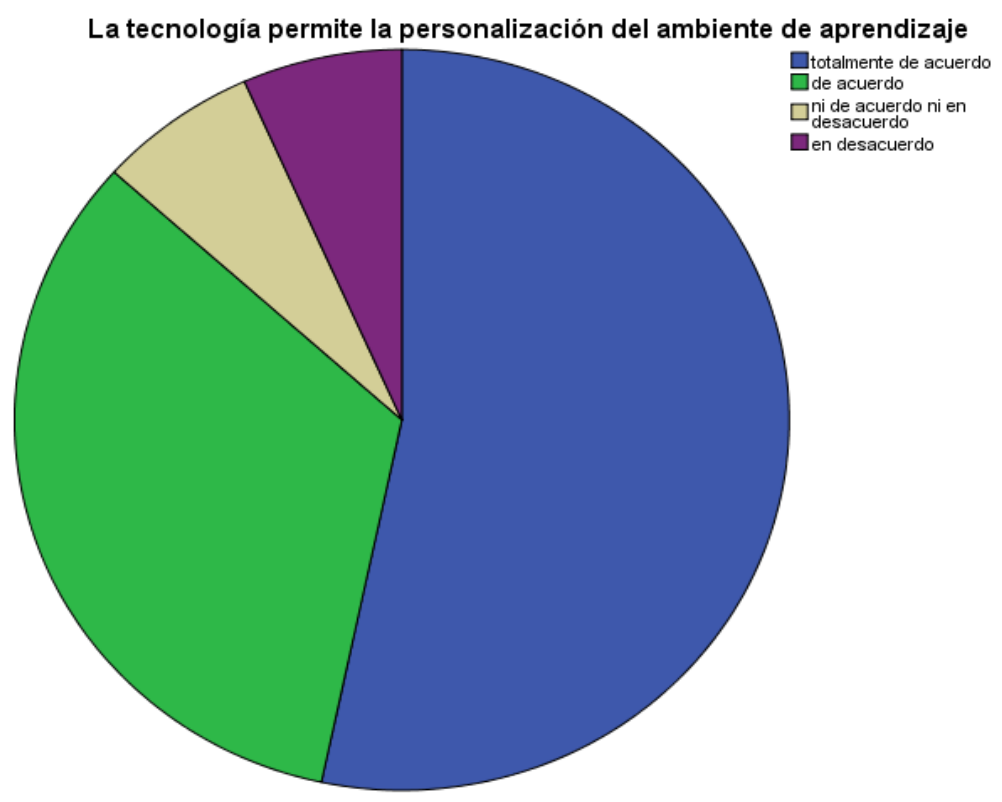

Fuente: Elaboración propia

El $86.7 \%$ de los estudiantes considera que la tecnología permite aprender de manera individual y didáctica en ambientes de aprendizaje, y únicamente el $6.7 \%$ está en desacuerdo.

Figura 9. El contenido generador de discriminación

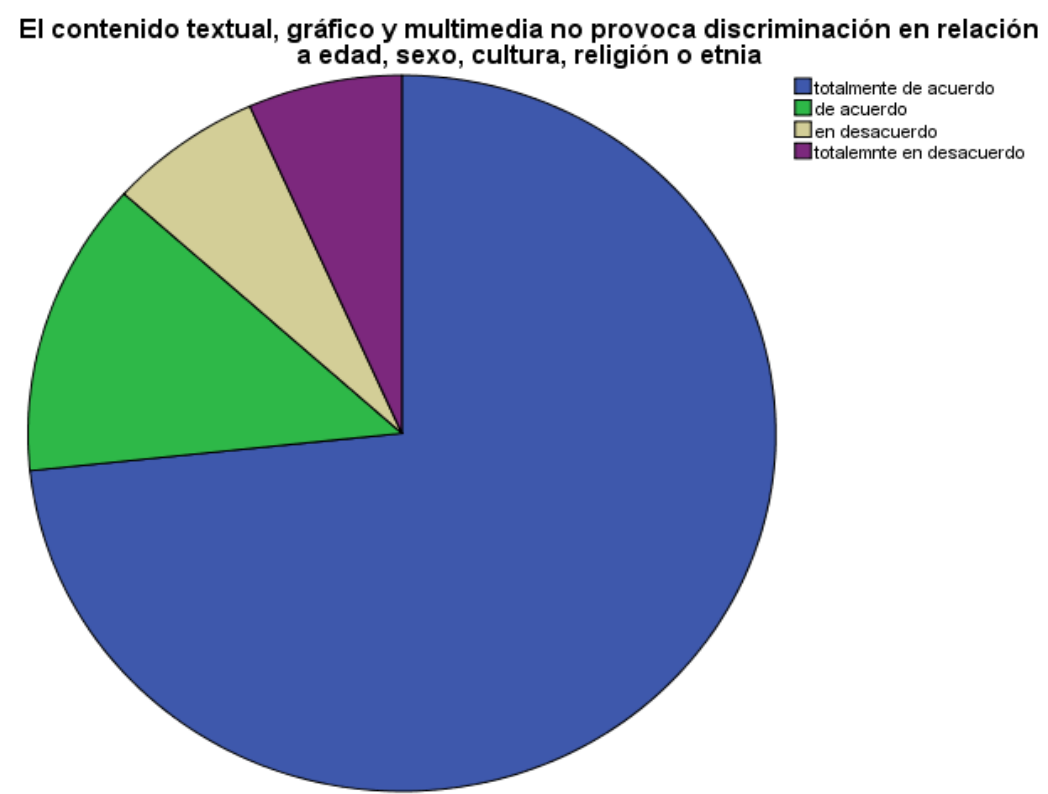

Fuente: Elaboración propia 
Se obtuvo un resultado porcentual del 73.3 con relación al contenido textual, gráfico y multimedia que no provoca discriminación de edad, sexo, cultura, religión o etnia comparada con el $6.7 \%$ que está totalmente en desacuerdo.

Figura 10. Contextualización del contenido

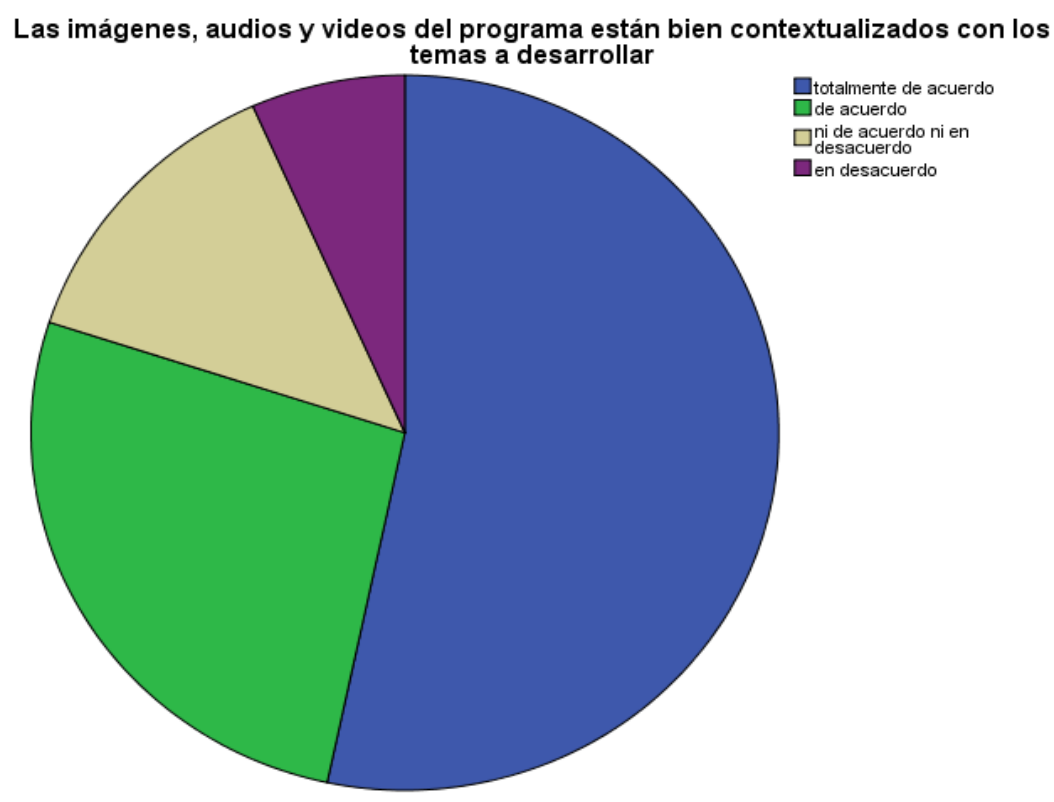

Fuente: Elaboración propia

El 80\% de la población encuestada está de acuerdo y totalmente de acuerdo en que los medios digitales son acordes con los temas a desarrollar en el programa.

Figura 11. Herramientas tecnológicas

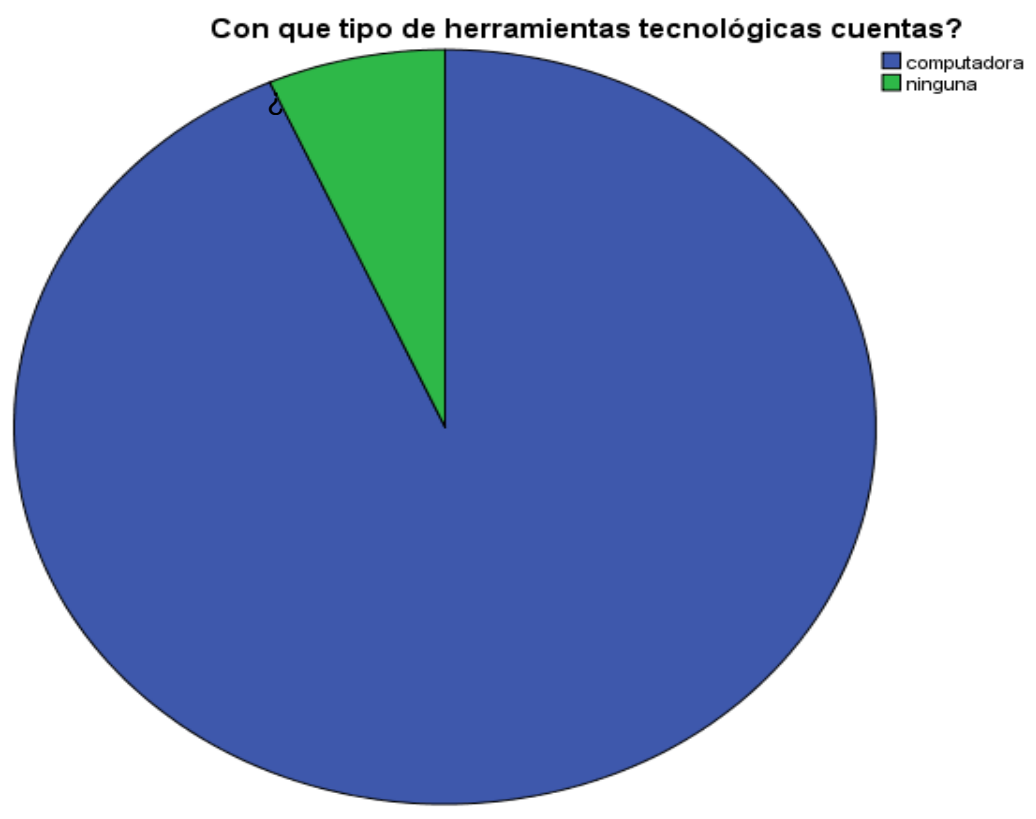

Fuente: Elaboración propia 
El $93.3 \%$ de los encuestados cuentan con computadora y solo el $6.7 \%$ no cuenta con ninguna herramienta tecnológica para uso personal y enfocado a la escuela.

Figura 12. Acceso de los estudiantes a internet

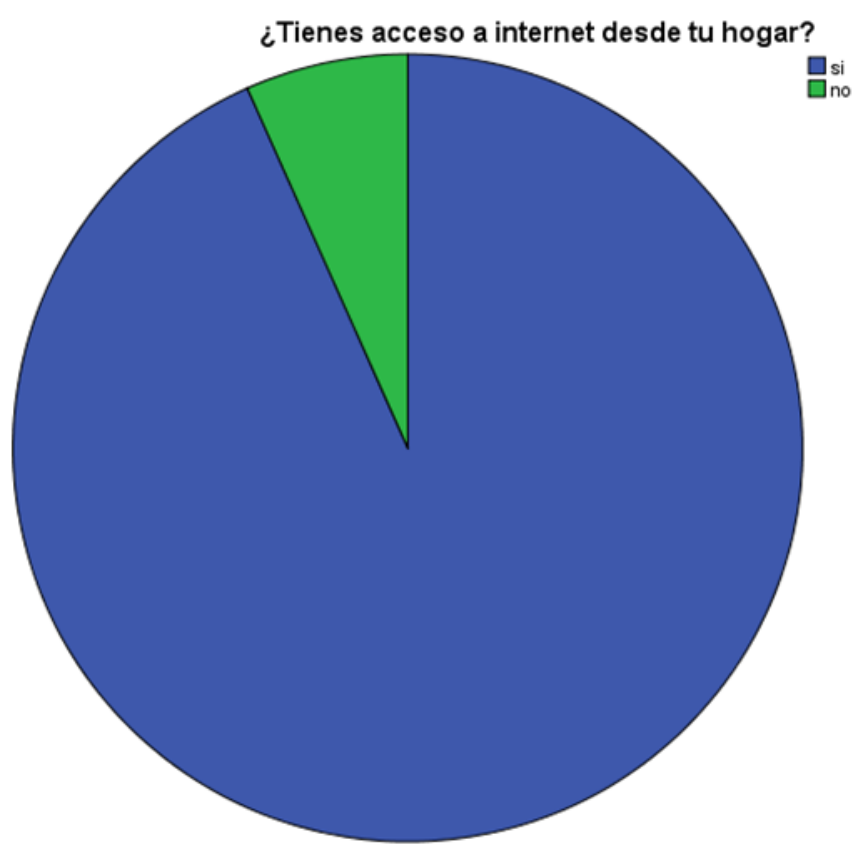

Fuente: Elaboración propia

El $93.3 \%$ de los encuestados si cuentan con acceso a internet y el $6.7 \%$ no tienen accesibilidad a internet para poder utilizarlo en sus tareas y trabajos.

Figura 13. Asignatura a distancia

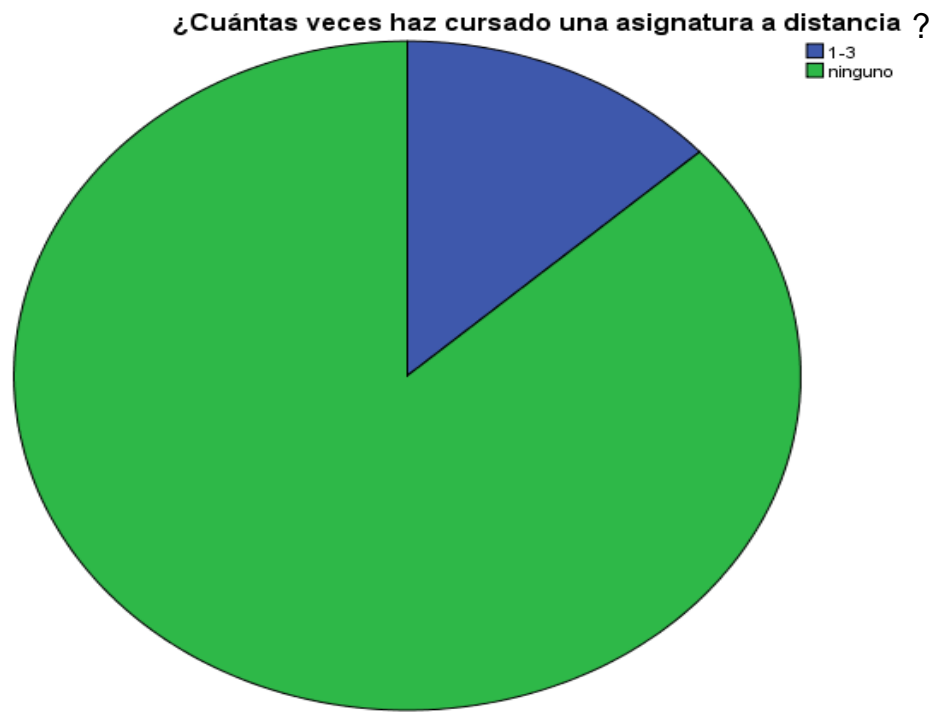

Fuente: Elaboración propia 
El $13.3 \%$ ha tomado de 1 a 3 veces asignaturas a distancia y el $86.7 \%$ no ha tomado ninguna asignatura a distancia, por lo tanto será un proceso más complejo introducirlos a este modelo.

Figura 14. Facilidad de un curso a distancia

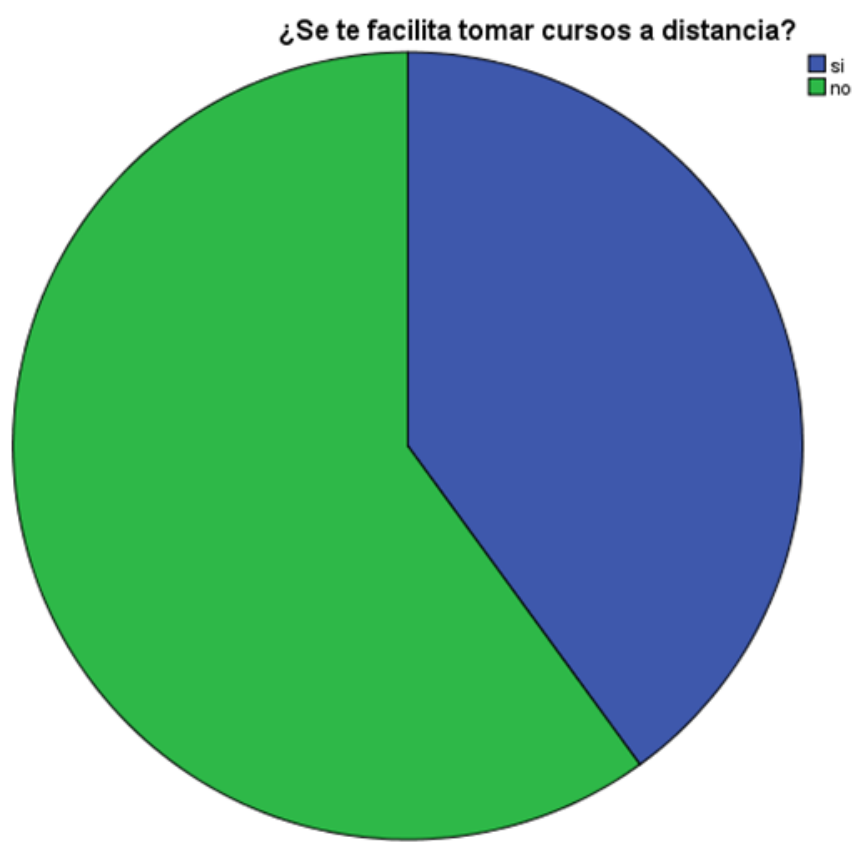

Fuente: Elaboración propia

El $60 \%$ de los alumnos no se le es fácil tomar cursos a distancia ya que no tienen experiencia, el $40 \%$ si se le facilitan los cursos, ya que, han podido experimentar las clases a distancia. 


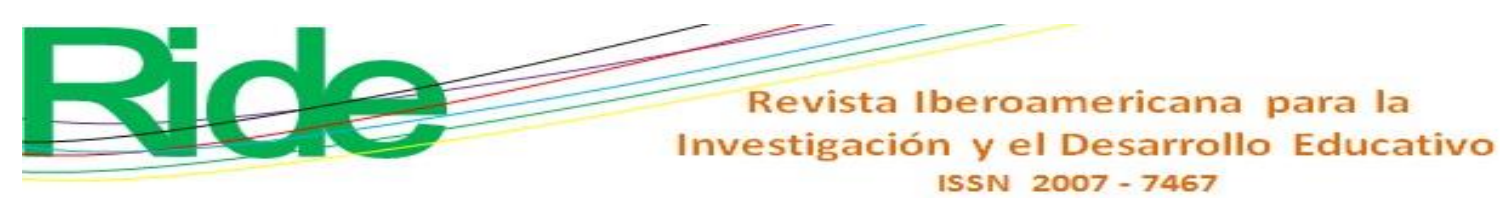

Figura 15. Viabilidad de asignatura a distancia

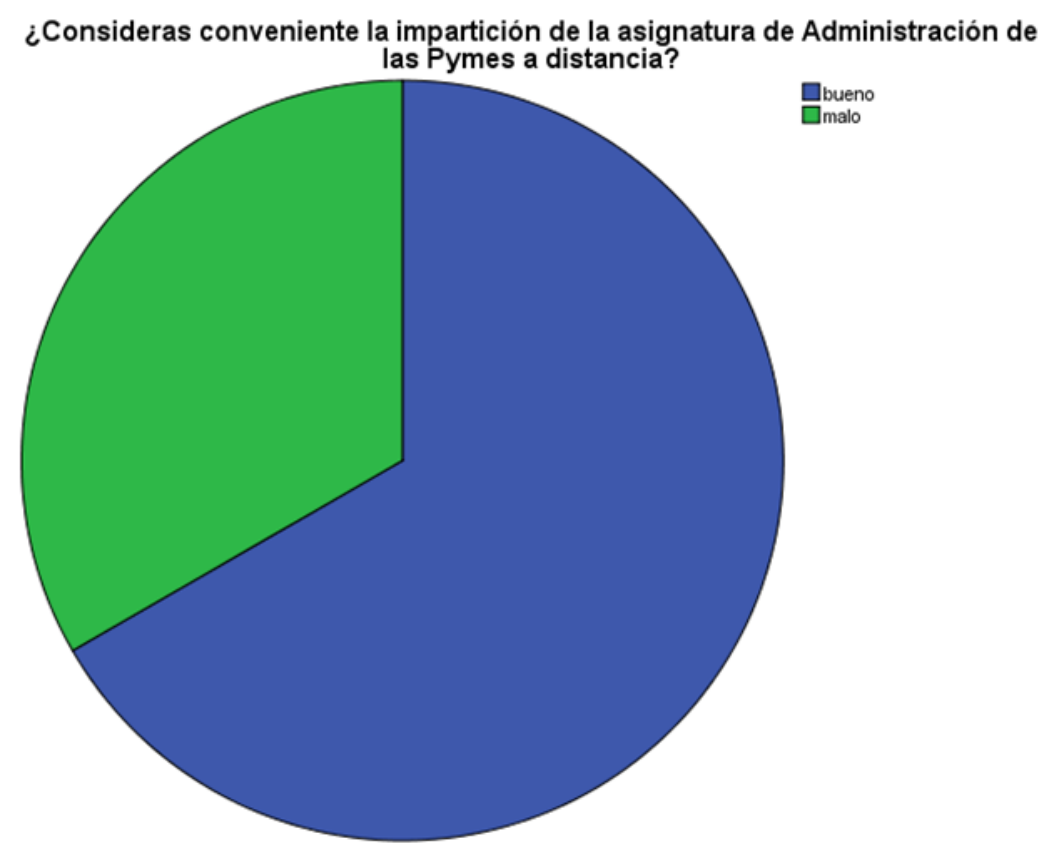

Fuente: Elaboración propia

El 66.7\% considera que si es bueno la impartición de la asignatura a distancia, y el 33.3\% considera mala la implementación de la asignatura a distancia, ya que no están acostumbrados a los cambios educativos y tecnológicos.

Los resultados obtenidos, considerando que la tecnología permite aprender de manera individual y didáctica en ambientes de aprendizaje, es elevada ya que los estudiantes consideran que se pueden utilizar las TIC's como una herramienta para mejorar el proceso de enseñanza-aprendizaje, teniendo como eje principal la comunicación guiada y orientada hacia el aprendizaje en la interacción existente entre docente-alumno.

Para estar en posibilidades de concretar los mecanismos de educación a distancia las universidades públicas se enfrenta a retos como la estructura curricular, la planeación, la estructuración de cursos, así como de programas educativos, es ahí donde participan los diferentes agentes, quienes conforman los proyectos institucionales para ofertar una educación a distancia de calidad. En este modelo educativo a distancia, es necesario contar con diferentes actores como son los propios coordinadores de los planes curriculares que de manera virtual determinaran las acciones y actividades a realizar por parte de los estudiantes, de igual forma estos coordinadores de proyectos universitarios, planean, capacitan, y estructuran la plantilla de docentes que intervendrán en este proceso educativo, así como la evaluación de los procesos educativos y su seguimiento. 


\section{Discusión}

La muestra estuvo conformada por 28 estudiantes de las Licenciaturas de Contaduría y Administración que cursaron la Unidad de Aprendizaje Administración de las PyMES, con un rango de edades de entre 19 y 43 años, contestando el cuestionario correspondiente a la investigación sobre la importancia de la implementación de la aplicación de la enseñanza a distancia de la UA, además de indagar si cuentan con computadora e internet para cursarla y las capacidades de los docentes para impartir la materia a distancia.

Se considera que en la actualidad el desarrollo de competencias, habilidades, aptitudes, actitudes, talentos y destrezas, generan el éxito de los profesionistas integrados al campo laboral, ya que las sociedades han ido evolucionando en la transformación de la información y el conocimiento, con la ayuda de las herramientas tecnológicas. Ya que la implementación de la tecnología ayuda a la vida en sociedad, facilitando los procesos y desarrollo de soluciones sociales.

El reto en las universidades es cada vez mayor, con la transformación de las nuevas sociedades de la información y el conocimiento, en la cual está la educación y la universidad inmersa en ella, (Casas, 2002), impulsando los cambios en los procesos y teorías de innovación, desarrollo y renovación, para ofrecer calidad social y profesional entre profesionistas.

Existiendo problemas con la implementación de la tecnología en las instituciones educativas públicas, por la falta de infraestructura, la mala calidad de la conectividad, erradicando los falsos problemas, para que el alumno pueda lograr con éxito no solo en el ámbito educativo sino global en su vida, ya que el alumno no es un ente aislado de la sociedad.

Fomentando la implementación de la utilización de las herramientas tecnológicas en los programas de clase, donde se elaboren apoyos didácticos de calidad, capacitando a los docentes en el manejo de plataformas educativas, instrumentos, bases de datos, entre muchos otros que existen. Interactuando de forma activa profesor-alumno en este proceso, donde el primero solo es facilitador y guía. Contando con una comunicación activa y adecuada, respetuosa, directa e imparcial.

Las TICs pueden contribuir al acceso universal a la educación. La UNESCO aplica estrategias de integración en la promoción de las TICs en la educación y el aprendizaje potenciado en tres sectores: comunicación e información, educación y ciencias. La tecnología de la educación es la suma total de las actividades que hacen que la persona modifique sus ambientes externos (materiales) o internos (de comportamiento). No hay 


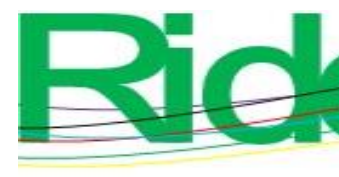

Revista Iberoamericana para la Investigación y el Desarrollo Educativo ISSN 2007 - 7467

que confundir máquinas o artefactos con aplicación de tecnologías, que tienen más que ver con la organización de medios y recursos que con los mismos medios. En el uso de la tecnología y los programas educativos necesitan ser apreciados en términos de eficacia y flexibilidad de aplicación en cuanto a tiempo, personal y recursos de que se disponga para que pueda ser empleada la tecnología en su hacer.

Se tiene que entender que como docente se deben buscar herramientas para la optimización del aprendizaje de los alumnos utilizando estrategias que ayuden a la solución de problemas contextuales reales, ayudados por las herramientas tecnológicas que puedan reforzar el conocimiento, con la ayuda de videos, foros de discusión, debates, lecturas, simuladores, audios, entre otros.

\section{Conclusiones}

Las conclusiones se presentan sobre los hallazgos encontrados en el estudio con relación a los objetivos, la información descriptiva sobre los participantes y para dar respuestas a las preguntas de investigación sobre la posibilidad de que los estudiantes consideren pertinente cursar la UA Administración de las PyMES de manera semipresencial, el 66.7 $\%$ de los encuestados consideran que sí es conveniente la impartición de la asignatura PyMES a distancia.

La educación a distancia requiere que los alumnos se encuentren dotados de estructura tecnológica, ya que el $86.7 \%$ consideran que las tecnologías de la información y comunicación son un medio accesible que favorecen a su formación. Asimismo, el 66.7\% consideran que sí es conveniente la impartición de la asignatura PyMES a distancia, sin embargo, existe un porcentaje que no está de acuerdo con ello, lo que quizá se deba a que no cuentan con el equipo tecnológico para cursarla.

La situación que se vive actualmente en el medio educativo ha mostrado que no en todas las familias cuentan con equipo suficiente para cubrir las necesidades de los integrantes que cursan algún nivel educativo.

Para la implementación de una educación semipresencial se requiere que participen además de los alumnos y las docentes que imparten la Unidad de aprendizaje, otros sujetos como un asesor que será quien guíe de manera específica el trayecto de los alumnos universitarios por esta Institución, orientarlos acerca de las alternativas técnicas y procesos de aprendizaje con el fin de cumplir con la meta propuesta; asimismo se requiere dotar al estudiante de herramientas didácticas. 
El guía o asesor deberá contar con características como empatía, respeto, solidaridad, así como con valores que fomenten un ambiente agradable y de confianza respecto a los alumnos.

Los docentes identificarán las características del estudiante más allá de las fronteras geográficas, y asumiendo diferentes contextos, en este sentido es necesario que el tutor se capacite y aplique de manera constante la práctica de nuevas estrategias y técnicas en los sistemas a distancia. Lo cual evaluará la actitud de los estudiantes hacia los nuevos paradigmas de las TIC's, conocer bien a sus alumnos. Ofrecer orientación e información. Evitar que se sienta aislado. Integrar los contenidos del curso a los objetivos propuestos. Ayudar al estudiante en el manejo de la tecnología. Apoyarlo a que se proponga metas. Motivar la comunicación y participación, determinando las siguientes funciones que deberá cumplir el tutor: proporcionar apoyo, motivación y orientación a los participantes, creando un ambiente ameno y estimulante.

Para apoyarlos en el manejo de la tecnología el guía o docente debe contar con los conocimientos y habilidades necesarias en el manejo de la tecnología, ya que como se ha podido observar en la situación actual que se está viviendo de manera extraordinaria, debido a la situación sanitaria, sin generalizar, los docentes no cuentan con la capacitación necesaria para enfrentar la situación de impartir clases a distancia.

Para que la educación dé verdaderos frutos, deben de contemplar la figura de los tutores, los cuales tienen como función principal realizar un acompañamiento efectivo de los alumnos, a fin de que exista una relación de cordialidad y empatía, lo que generará una atmosfera propicia para que los estudiantes lleven a cabo las tareas asignadas, en este sentido los tutores tienen una gran responsabilidad, por lo que es necesaria la capacitación y actualización constante, es de señalar que los tutores deben advertir, además de diagnosticar problemáticas de carácter académico, orientar de manera completa a los estudiantes y efectiva en la elección de su situación académica.

Los docentes, asesores, tutores y el soporte técnico en la educación a distancia deberán ser responsables de organizar, estructurar, y direccionar los programas de tutorías en las instituciones de educación superior, con el propósito de atender las necesidades académicas de los universitarios, incorporando las nuevas tecnologías a los programas tutoriales. Siendo persona capaz y profesional, que atiende, orienta y plantea escenarios académicos en los cuales orienta a los estudiantes en la conformación de sus procesos cognitivos de manera integral, estableciendo una serie de canales, con el propósito de que tengan una forma didáctica y efectiva de hábitos de estudio, capaz de integral una serie de estrategias de aprendizaje, para que el discente aproveche su estadía en las 


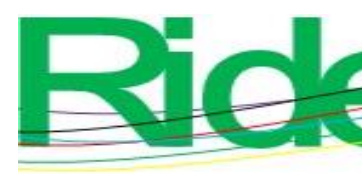

Revista Iberoamericana para la Investigación y el Desarrollo Educativo ISSN $2007-7467$

instituciones educativas. También plantea alternativas para exponer contenidos significativos de acuerdo con las temáticas desarrolladas en las unidades de aprendizaje de los universitarios, establece la planeación de los cursos, actividades, así como la capacitación en entornos virtuales, y diseño multimedia.

El óptimo desarrollo de la educación a distancia, se complementa con la utilización de herramientas electrónicas que cubran las necesidades de los estudiantes, lo que significa que deben de existir elementos actuales como equipos de cómputo, software interactivo, portales accesibles, nuevas tecnologías, que den soporte para la realización de las tareas de los estudiantes; las universidades deben tener en consideración que las herramientas tecnológicas deben tener un soporte innovador, con la posibilidad de que participen de manera activa así como la alternativa de interactuar de manera directa no solo con los asesores, tutores, sino con la comunidad educativa en la cual se desempeñan.

Con la triple hélice en donde el alumno es el principal actor además de la ayuda de facilitadores y tutores se complementa esta integración de aprendizaje, donde el actor principal administra su tiempo y las herramientas con las que cuenta además de implementar el proceso administrativo (planeación, organización, dirección y control) al máximo, se debe poseer el gusto por la lectura y la redacción, asimismo tener un sentido crítico por la autoeducación.

\section{Futuras líneas de investigación}

Los resultados de la investigación, así como el análisis documental sobre la misma, sugieren una serie de líneas de investigación futura sobre lo relacionado con la formación en el Centro Universitario UAEM Ecatepec, inovando en los planes o programas de estudio, actualizaciones en el personal de docencia en la utilización de herramientas tecnológicas para su utilización en la docencia, implementar o actualizar los programas de estudio de acuerdo a la demanda del mundo laboral, investigar las competencias profesionales, valores y actitudes de los alumnos, así como los recursos con los que cuenta o podría contar y su situación económica, dificultades que presentaron los alumnos al cursar las materias en línea, al termino del curso verificar si se cumplió el objetivo del curso en cuanto al aprendizaje obtenido por parte de los alumnos. 


\section{Referencias}

Cordera Campos, R. (2008). La autonomía en la sociedad del conocimiento. Recuperado el 2018, de Universidades: https://www.redalyc.org/pdf/373/37303803.pdf

Díaz, H. (2014). Las Ciencias Sociales en la Sociedad del Conocimiento. Recuperado el 2018, de Diálogo Andino- Revista de Historia, Geografia y Cultura Andina. Universidad de Tarapacá: http://www.redalyc.org/articulo.oa?id=371333938001

García, F. P. (2001). Nativos digitales y modelos de aprendizaje. Recuperado el 26 de 09 de 2020, de http://ceur-ws.org/Vol-318/Garcia.pdf

Gutiérrez, A. (1997). Integración Curricular de las TIC’s y Educación para los Medios en la Sociedad del Conocimeinto. España: Edicones de la Torre.

Henríquez, P. (2001). La Aplicación didactica de las Tecnologías de la Informatica y la Comunicación en la formación del siglo XXI. Recuperado el 2018, de Tesis Doctoral, Universidad Rovira I Virgili. Tarragona.

Holmberg, B. \&. (s.f.). Guided didactic conversation in distance education. En Distance Education: International Perspectives (págs. 114-122). New York.

México, U. A. (2009). Plan General de Desarrollo 2009-2021. Recuperado el 2020, de Universidad Autónoma del Estado de México: http://web.uaemex.mx/abogado/doc/0057\%20LinEdCont.pdf

Ortega, F. (2007). ¿Una sociedad del conocimeinto sin intelectuales? Recuperado el 2017, de Universidad de los Hemisterios: http://www.redalyc.org/articulo.oa?id=199520735006

Salinas, J. (2004). Hacia un modelo de educación flexible: Elementos y reflexiones. Madrid, España: Pearson-Prentice Hall.

Sangrá, A. (2003). La integración de las TIC’s a la Universidad: una aproximación estrategica. Proyecto de investigación. Univerdiad Oberta de Catalunya, Barcelona.

Torres, S. A. (2012). ¿Hacia una sociedad del conocimiento? consideraciones a partir del desarrollo de la ciencia, la educación superior y las TIC's. Recuperado el 2019, de

https://www.facebook.com/sharer/sharer.php?u=https\%3A\%2F\%2Fredie.uabc.m x\%2Findex.php\%2Fredie\%2Farticle\%2Fview\%2F307\%2F662

UNESCO. (1998). Declaración mundial sobre la educación superior en el siglo XXI: Visión, acción y marco de acción prioritaria para el cambio y el desarrollo de la educación superior. Recuperado el 2019, de http://www.unesco.org/education/educprog/wche/declaration_spa.htm 


\begin{tabular}{|l|l|}
\hline Rol de Contribución & Autor (es) \\
\hline Conceptualización & Patricia Delgadillo Gómez \\
\hline Metodología & Leisdy Del Carmen Gutiérrez Olmos \\
\hline Software & Patricia Delgadillo Gómez \\
\hline Validación & Adriana Mercedes Ruiz Reynoso \\
\hline Análisis Formal & Sara Lilia García Pérez \\
\hline Investigación & Leisdy Del Carmen Gutiérrez Olmos \\
\hline Recursos & Matilde Gómez Méndez \\
\hline Curación de datos & Adriana Mercedes Ruiz Reynoso \\
\hline $\begin{array}{l}\text { Escritura - Preparación del } \\
\text { borrador original }\end{array}$ & Matilde Gómez Méndez \\
\hline $\begin{array}{l}\text { Escritura - Revisión y } \\
\text { edición }\end{array}$ & Matilde Gómez Méndez \\
\hline Visualización & Sara Lilia García Pérez \\
\hline Supervisión & Leisdy Del Carmen Gutiérrez Olmos \\
\hline Administración de Proyectos & Patricia Delgadillo Gómez \\
\hline Adquisición de fondos & Adriana Mercedes Ruiz Reynoso \\
\hline
\end{tabular}

